\title{
PROPOSTA DE UM MODELO PARCIMONIOSO ENTRE A EMPATIA E ORIENTAÇÃO CULTURAL EM BRASILEIROS
} A PROPOSAL FOR A PARSIMONIOUS MODEL BETWEEN EMPATHY AND CULTURAL ORIENTATION TYPE IN THE BRAZILIAN POPULATION

Recibido: 01 de Abril del 2013 | Aceptado: 09 de Julio del 2013

NILTON S. FORMIGA

(FACULDADE MAURICIO DE NASSAU, João Pessoa, PB, Brasil)

\begin{abstract}
RESUMO
$\mathrm{Na}$ sociedade contemporânea é possível observar constantes mudanças nos espaços sociais, econômicas e culturais ocorrendo na vida das pessoas. Tais mudanças podem-se referir a orientação individualista e coletivista que as aderem na dinâmica do seu entorno psicossocial. Acredita-se que estas orientações não surgem do vazio social, mas, a partir do reconhecimento da pessoa de sua capacidade de ressonância interpessoal, isto é, de empatia. 0 presente estudo tem como objetivo verificar a associação da empatia sobre orientação cultural de individualismo e coletivismo. Para isso foram aplicadas a escala multidimensional de reatividade interpessoal de Davis, a escala dos atributos do tipo de orientação cultural individualista e coletivista e dados sócio-demográficos em 382 pessoas do sexo masculino e do sexo feminino, com idades entre 14 a 61 anos. Em pessoas que estudam em escolas privadas e públicas das cidades de João Pessoa-PB e Natal-RN. A análise da modelagem estrutural apresentou uma forte associação positiva da empatia com 0 tipo de orientação coletivista e uma fraca associação com a orientação individualista. Como resultado identificamos a importância de se desenvolver práticas potencializadoras das habilidades empáticas a fim de gerar ações culturais que valorizem 0 outro e a tradição.
\end{abstract}

PALAVRAS CHAVE: Empatia, Atributos individualismo-coletivismo, Modelo teórico.

\section{ABSTRACT}

Constant changes in people's social, economic, and cultural lives are seen in contemporary society. These changes may be related to the individualistic and collectivist orientations that are linked in the dynamics of the psychosocial environment. These orientations do not arise from a social vacuum, but from the recognition by the person of his capacity for empathy. The present study aims to identify the relationship between empathy and the cultural orientations of individualism and collectivism. We applied the multidimensional scales of the Davis Interpersonal Reactivity Index regarding the type of individualist and collectivist cultural orientations and socio-demographic data on 382 men and women. All subjects were between the ages of 14-61 and studying in public and private schools in the cities of João Pessoa and Natal-RN in Brazil. The structural model analysis showed a strong positive association of empathy with the collectivist orientation type and a weak association with individualistic orientation type. As a result we have identified the importance of developing a series of practices that promote empathy skills to generate cultural activities that promote valuing others and traditions

KEY WORDS: Empathy, Individualism-collectivism attributes, Theoretical model. 


\section{INTRODUÇÃO}

El No desenvolver da sociedade contemporânea é possível acompanhar a ocorrência de um conjunto de mudanças (mudança social, econômica e cultura) suscitando melhorias na dinâmica interpessoal e ambiental. Tal condição tem modificado o processo do significado e sentido da vida indivíduo-sociedade em relação ao estabelecimento de um modelo de pensamento e conduta socialmente desejável, moral e ética.

Apesar dessas mudanças serem representadas como uma forma positiva na funcionalidade e estrutura psicológica e social das pessoas, observa-se a existência de uma sociedade que tem investido cada vez mais na experiência ambivalente, tendenciosa ao risco e focada na satisfação exacerbada do sujeito em si mesmo (Bauman, 1998; Burger, 1985; Howard, 2000; Jodelet, 1984; Kumar, 1997; Lipovetsky \& Charles, 2004; Teixeira, 2005). De acordo com Formiga e Souza (2012), contextualmente, tem sido identificada na existência dos conflitos entre a conduta grupal e individual, a valoração pelo outro e 0 fingimento cooperativo, etc., uma aderência a condutas mais individualistas e utilitaristas, manifestando um baixo vinculo no cuidado e da valoração humana.

Essas condições sugerem que, se 0 comportamento das pessoas depende de como elas percebem a situação em que se encontram, é possível que, a partir de tal apercepção, elas definam a sua própria realidade na relação psicossocial com os outros (Atteslander, 1999). Isto é, a pessoa passaria a se orientar culturalmente, através de crenças, valores, atitudes, etc., se somente se, a partir de uma disposição que elas venham a ter quanto às trocas de experiências, cognitiva e afetiva, no desenvolvimento da empatia, sendo capaz de reconhecer uma ressonância interpessoal em direção de uma conduta socialmente aceita.
Neste sentido, o psiquismo humano poderá ser conduzido, seja o psiquismo de quem precisa de ajuda, seja daquele que ajuda (ou melhor, de quem se empatiza), a uma disposição ao acolhimento dos outros e o desdobramento de horizontes de sentidos da situação que se experiencia, reabrindo espaço para interação humana e seus vínculos afetivos (Formiga \& Souza, 2012). Tendo por base 0 desenvolvimento da empatia, o sujeito passaria a se orientar culturalmente, seja de forma individualista ou coletivista, em direção ao outro e, na medida em que fossem afloradas melhores habilidades empáticas, maior seria a condição de que a pessoa se orientasse de forma que se preocupasse mais com os outros, compartilhando seus interesses e cooperando ao invés de pensar somente em si.

Ao enfatizar essa orientação cultural, faz-se referência aos padrões convencionais adotado por cada pessoa; trata-se da priorização de atributos dos valores culturais, reconhecidos como valores de individualismo e coletivismo, capaz de orientar as escolhas e as atitudes humanas (Rokeach, 1973; 1979; Triandis, 1995; 1996). Neste contexto, ao considerar estes atributos como padrões culturais, 0 sujeito poderá adotar uma ação individualista, apresentado uma tendência ao sucesso, a valorização da própria intimidade e uma necessidade de adequarse ao contexto social, visando obter recompensas; ou poderá atuar de forma coletivista, tendendo a cooperação e ao cumprimento com relação aos demais, bem como, manter fortes relações entre si, podendo compartilhar os mesmos interesses (Gouveia, Clemente \& Vidal, 1998).

Com isso, o individualismo e coletivismo são definidos como síndromes culturais e consistem em que as pessoas compartilhem atitudes, crenças, normas, papéis sociais e definições do eu (Triandis, 1995; 1996). Sendo assim, as pessoas que se orientam 
por um tipo ou outro de orientação cultural, vão se comportar de maneira diferente, seja na forma de se auto-perceber ou nos relacionamentos interpessoais. Triandis (1995), com base na perceptiva teórica de Hofstede (1980), o qual, ao avaliar as dimensões culturais nas empresas, enfatiza o papel do sujeito ou do grupo na dinâmica das sociedades humanas, identificando os atributos horizontal (sugere que as pessoas são similares na maioria dos aspectos, especialmente no status) e vertical (enfatiza a aceitação da desigualdade e privilegiar a hierarquia) para diferenciar os tipos de individualismo e coletivismo.

Tais atributos se combinam com 0 individualismo e coletivismo formando quatro tipos de orientação, cada um com uma característica principal que melhor descreve a pessoa que adota cada um destes tipos, a saber: Individualismo Horizontal (refere-se a um Ser único, diferente dos demais) e o Vertical (salienta a Orientação ao êxito, ao triunfo); enquanto 0 coletivismo Horizontal (refere-se a pessoa Cooperadora, que colabora) e o Vertical (diz respeito ao sujeito Cumpridor com os demais, ser servidor). De acordo com Formiga (2004; Formiga \& Diniz, 2011), estes atributos do individualismo e do coletivismo podem se relacionar a uma variedade de fatos e pensamentos nas múltiplas facetas da vida social e política (Inglehart, 1991).

Desta forma, reflete-se: como é possível e por qual variável psicológica tais atributos poderiam se originar no sujeito? Acredita-se que os atributos existiriam a partir do desenvolvimento de habilidades empáticas, já que esta se refere a uma disposição funcional das pessoas para as trocas de experiências expostas, incondicionalmente, em relação ao outro, podendo ser definida como uma resposta afetiva de origem evolutiva, mais apropriada à situação do outro do que da própria pessoa (Decety, 2005; Decety \& Jackson, 2004; Decety,
Michalska \& Akitsuki, 2008; Enz \& Zoll, 2006; Wispé, 1990).

Com isso, uma pessoa empática, teoricamente, é capaz de experimentar vicariamente emoções sentidas por outra pessoa, adotar o ponto de vista do outro, compreender suas motivações e necessidades e atribuir atitudes e comportamentos ao outro com a função de prover ajuda, agregação, cuidado, justiça e solidariedade (Batson, Eklund, Chermok, Hoyt \& Ortiz, 2007; Batson, Tricia, Highberger \& Shaw, 1995; Davis 1983; Hoffman, 2000; Mehrabian \& Epstein, 1972).

De acordo com a perspectiva psicogenética, evolutiva e multidimensional da empatia proposta por Davis (1983), as habilidades empáticas são distribuídas em quatro construtos independentes, os quais avaliam experiências afetivas e cognitivas da pessoa: quanto à experiência cognitiva, destaca-se 0 construto da tomada de perspectiva do outro (refere-se à capacidade cognitiva voltada para a compreensão e coordenação de percepções do outro que visem à solução de conflitos interpessoais e sociais); e da fantasia (refere-se a habilidade de se identificar com personagens ficcionais em novelas, filmes e romances e sentir junto com eles, uma adesão involuntária às condições afetivas de alegria, tristeza, raiva etc. e/ou de necessidade destes personagens).

No que diz respeito à experiência afetiva, as pessoas poderão acessar tais habilidades através da consideração empática (diz respeito à capacidade de avaliar e sentir com o outro, bem como do reconhecer seus afetos e necessidades, que pode ser experimentada no self como uma motivação de cunho pró-social que pode levar ao comportamento de ajuda) e da angustia Pessoal (refere-se a um sentimento de tensão e desconforto, frente à condição de necessidade do outro, podendo gerar comportamentos de 
afastamento ao invés de comportamentos de ajuda).

Partindo dessas reflexões, o presente estudo tem como objetivo a verificação de um modelo teórico parcimonioso, contrário ao que salientou Formiga e Souza (2012). Para esses autores, a orientação cultural coletivista influencia, positivamente, a empatia; enquanto a orientação individualista influencia, negativamente, a empatia. Mas, no seu estudo, observou-se no resultado um problema que poderá ser destacado com base na perspectiva teórica, metodológica e estatística:

Segundo Formiga e Souza (2012), o sujeito, primeiro, se orienta culturalmente para depois desenvolver a empatia. Refletese, no presente estudo, uma outra direção: sendo a empatia um construto psicogenético, evolutivo e multidimensional (Davis, 1983) podendo ser avaliado no seu conjunto geral (como empatia geral), mas, também, em dimensões específicas (por exemplo, consideração empática, tomada de perspectiva do outro, angustia Pessoal e fantasia), esta, poderá associar-se a orientação cultural - coletivista ou individualista - positivamente, a partir da dinâmica dessas habilidades empáticas.

E mais, não é possível que a empatia esteja associada negativamente, a um destes atributos, pois, eles poderão ser assimilados um pouco de cada um pelas pessoas, os quais têm apenas o contexto como controle do estilo cultural que melhor possa ser manifestado, não existindo oposição entre eles (Sinha \& Tripathi, 1994; Schwartz, 1990; Triandis, 1995; Triandis, Chen \& Chan, 1998); desta forma, é possível que a maior aderência do sujeito a um ou outro desse atributo seja predito pela habilidade empática e não o inverso como enfatiza Formiga e Souza (2012).

Teoricamente, o que vai influenciar é a capacidade que 0 sujeito possa ter quanto ao seu desenvolvimento na empatia, pois, tendo assimilado qualitativamente tal construto, provavelmente, será menor a associação com a orientação cultural individualista e maior com a orientação cultural coletivista. Em termos estatísticos, 0 estudo de Formiga e Souza (2012) revelam indicadores estatísticos do modelo, por exemplo, o qui-quadrado com grau de liberdade $\left(\chi^{2} / \mathrm{gl}\right)$ que foi abaixo de 1.00, condição que sugere um excesso nos ajustes dos erros colocando o modelo proposto pelos autores supracitados em uma interpretação que merece certo cuidado para explicar a direção teórica que estes defendem.

Partindo desses pressupostos, este estudo tem como objetivo verificar, a partir da análise e modelagem de equação estrutural no programa AMOS 16.0, a associação da empatia (tanto em seu construto geral quanto em suas dimensões especificas) com 0 tipo de orientação cultural coletivista e individualista. Hipoteticamente, espera-se que a empatia geral, bem como, as suas dimensões empáticas, se associem mais com mais força relacional com a orientação coletivista e mais fraca com a individualista.

\section{MÉTODO}

\section{Amostra}

382 sujeitos, do sexo masculino $(40 \%)$ e do sexo feminino $(60 \%)$, de 14 a 61 anos ( $\mathrm{M}=$ 25,96 ; d.p. $=12,04$ ) compuseram este estudo. Estes foram do nível fundamental, médio e universitário de instituições privadas e de publicas das cidades de João Pessoa-PB (53\%) e Natal - RN (47\%). A amostra foi não probabilística, pois considerou-se a pessoa que, consultado, se dispôs a colaborar, respondendo 0 questionário a ele apresentado, bem como, buscou-se garantir uma variabilidade dos resultados na distribuição das idades. 
Instrumentos

Escala Multidimensional de Reatividade Interpessoal de Davis - EMRI. Trata-se de um instrumento elaborado por Davis (1983) e adaptado em sua versão original por Sampaio, Guimarães, Camino, Formiga e Menezes (2011) para o contexto brasileiro. Um estudo posterior ao desses autores foi desenvolvido por Formiga (2012) verificando a acurácia do mesmo; esse autor observou indicadores psicométricos $\left(\chi^{2} / \mathrm{gl}=1,04, \mathrm{GFI}\right.$ $=0,98, \mathrm{AGFI}=0,96, \mathrm{RMR}=0,03, \mathrm{CFI}=$ $1,00, \operatorname{RMSEA}(90 \% \mathrm{IC})=0,00(0,00-0,02)$, $\mathrm{CAIC}=1208,51$ e ECVI $=0,76$ ) próximos aos encontrado por Sampaio e cols. (2011), os quais, além de corroborar o estudo, revelaram a manutenção da medida desse instrumento.

O instrumento é composto por 26 sentenças que descrevem comportamentos, sentimentos e características relacionadas à empatia, que são utilizadas para avaliar as dimensões de experiência cognitiva, como a tomada de perspectiva do outro e a fantasia; e de experiência afetiva composta pela consideração empática e angustia Pessoal. Todas as questões foram avaliadas por escalas likert, que variam de 1 ("não me descreve bem") a 5 ("descreve-me muito bem"). Escores mais altos indicam níveis mais elevados em cada uma dessas dimensões e a soma dos escores de todas as subescalas é utilizada para calcular 0 nível global de empatia. 0 item 2 (Sou neutro quando vejo filmes) deve ter sua pontuação invertida, pois foi elaborado na direção contrária a dos demais itens da escala.

Escala dos atributos do tipo de orientação cultural individualista e coletivista. Composto por seis itens que avaliam os atributos que mais caracterizam os sujeitos em relação ao individualismo e coletivismo (por exemplo, Um ser único, diferente dos demais; Orientado ao êxito, ao triunfo, Cooperador, que colabora; Cumpridor com os deveres, ser servidor). Formiga e Mota (2009) observaram Alfas de Cronbach aceitáveis pela literatura sobre o tema; realizando uma análise fatorial confirmatória (AFC) e a análise do modelo de equação estrutural (SEM), Formiga (2011) observou que este escala mostrou-se fidedigna quanto a estrutura fatorial já observada pelos autores supracitados, por exemplo: $\chi^{2} / g l=3,01$; GFI $=0,99$ e $\mathrm{AGFI}=0,97 ; \mathrm{TLI}=0,93 ;$ RMSEA $(90 \% \mid C)=0,05(0,03-0,08), C A I C=131,58$ e ECVI $=0,07$.

0 instrumento proposto apresentou garantia de maior confiabilidade fatorial e evidências empíricas para sua aplicação e mensuração no contexto paraibano. Para respondê-lo o jovem deveria ler cada item e indicar o quanto cada um dos atributos the caracteriza, para isso, era necessário apontar (com um X ou circulo) numa escala do tipo Likert, com os seguintes extremos: 0 = Nada Característico e $\mathbf{5}=$ Muito Característico, ao lado dos respectivos atributos.

Caracterização Sócio-Demográfica. Foram elaboradas perguntas que contribuíram para caracterizar os participantes deste estudo (por exemplo, sexo, idade, classe sócioeconômica), bem como, realizar um controle estatístico de algum atributo que possa interferir diretamente nos seus resultados.

\section{Procedimentos}

Todos os procedimentos adotados nesta pesquisa seguiram as orientações previstas na Resolução 196/96 do CNS e na Resolução 016/2000 do Conselho Federal de Psicologia (CNS, 1996; ANPEPP, 2000) sobre as pesquisas com seres humanos.

\section{Administração}

Colaboradores com experiência prévia na administração da aplicação dos instrumentos foram responsabilizados pela coleta dos dados, e apresentaram-se nas salas de aula como interessados em conhecer as opiniões e os comportamentos 
dos alunos sobre as situações descritas nos instrumentos.

Solicitou-se a colaboração voluntária dos jovens no sentido de responderem um breve questionário. Após ficarem cientes das condições de participação na pesquisa, assinaram um termo de Consentimento Livre e Esclarecido. Foi-lhes dito que não havia resposta certa ou errada. A todos foi assegurado 0 anonimato das suas respostas informando que estas seriam tratadas em seu conjunto. As escalas foram então respondidas individualmente.

Apesar de 0 instrumento ser autoaplicável, contando com as instruções necessárias para que possam ser respondidos, os colaboradores na aplicação estiveram presentes durante toda a aplicação para retirar eventuais dúvidas ou realizar esclarecimentos que se fizessem indispensáveis. Um tempo médio de 30 minutos foi suficiente para concluir essa atividade.

\section{Análise dos dados}

No que se refere à análise dos dados desta pesquisa, utilizou-se a versão 18.0 do pacote estatístico SPSS para Windows. Foram computadas estatísticas descritivas (tendência central e dispersão). Os seguintes indicadores estatísticos para 0 Modelo de Equações Estruturais (MEE) foram considerados segundo uma bondade de ajuste subjetiva. Esse programa estatístico tem a função de apresentar, de forma mais robusta, indicadores psicométricos que vise uma melhor construção da adaptação e acurácia da escala desenvolvida, bem como, permita desenhar um modelo teórico pretendido no estudo.

No que se refere à análise dos dados desta pesquisa, utilizou-se a versão 18.0 do pacote estatístico SPSS para Windows. Foram computadas estatísticas descritivas (tendência central e dispersão). Indicadores estatísticos para o Modelo de Equações Estruturais (SEM), efetuados no AMOS GRAFICS na versão 16.0, foram considerados segundo uma bondade de ajuste subjetiva:

- $0 \chi^{2}$ (qui-quadrado) testa a probabilidade de o modelo teórico se ajustar aos dados; quanto maior este valor pior 0 ajustamento. Este tem sido pouco empregado na literatura, sendo mais comum considerar sua razão em relação aos graus de liberdade $\left(\chi^{2} / g\right.$. .l.). Neste caso, valores até 5 indicam um ajustamento adequado;

- Root Mean Square Residual (RMR), que indica o ajustamento do modelo teórico aos dados, na medida em que a diferença entre os dois se aproxima de zero. Para o modelo ser considerado bem ajustado, 0 valor deve ser menor que 0,05;

- O Goodness-of-Fit Index (GFI) e o Adjusted Goodness-of-Fit Index (AGFI) são análogos ao $R^{2}$ em regressão múltipla. Portanto, indicam a proporção de variância-covariância nos dados explicada pelo modelo. Estes variam de 0 a 1 , com valores na casa dos 0,80 e 0,90, ou superior, indicando um ajustamento satisfatório (Hair, Anderson, Tatham \& Black, 2005; Bilich, Silva \& Ramos, 2006);

- A Root-Mean-Square Error of Approximation (RMSEA), com seu intervalo de confiança de $90 \%$ (IC90\%), é considerado um indicador de "maldade" de ajuste, isto é, valores altos indicam um modelo não ajustado. Assume-se como ideal que o RMSEA se situe entre 0,05 e 0,08 aceitandose valores de até 0,10 (Kelloway, 1998; Garson, 2003); 
- NFI, varia de zero a um e pode ser considerado aceitável para valores superiores a 0,90. Caracteriza-se por ser uma medida de comparação entre 0 modelo proposto e o modelo nulo, representando um ajuste incremental;

- O Comparative Fit Index (CFI) compara de forma geral 0 modelo estimado e o modelo nulo, considerando valores mais próximos de um como indicadores de ajustamento satisfatório (Joreskög \& Sörbom, 1989; Hair; Tatham; Anderson \& Black, 2005).

- Root Mean Square Residual (RMR), que indica o ajustamento do modelo teórico aos dados, na medida em que a diferença entre os dois se aproxima de zero. Para o modelo ser considerado bem ajustado, 0 valor deve ser menor que 0,05 .

\section{RESULTADOS E DISCUSSÃO}

Para atender o objetivo do presente estudo, considerou-se um modelo recursivo de equações estruturais; efetuou-se uma análise e modelagem de equação estrutural no programa AMOS 16.0, para os modelos que se pretendiam verificar. Sendo assim, inicialmente, procurou-se verificar esses modelos para as variáveis empatia (e suas variações) em relação a orientação coletivista e em seguida para a empatia e a orientação individualista; realizadas as devidas modificações nos ajustes de erro, encontraram-se modelos adequados, tanto para o conjunto geral das dimensões da empatia [isto é, composta pela consideração empática (CE), angústia pessoal (AP) e tomada de perspectiva (TP) e fantasia (FS)] associada a orientação cultural coletivista [Cooperador, que colabora e Cumpridor com os demais, ser servidor], bem como, para cada especificidade das dimensões da empatia (ver tabela 1). Estes modelos apresentaram indicadores psicométricos que estiveram dentro do intervalo aceito pela literatura estatística (Hair, Anderson, Tatham \& Black, 2005; Joreskög \& Sörbom, 1989) corroborando uma forte associação da empatia em relação ao tipo de orientação cultural coletivista. Porém, merece destacar a existência de indicadores psicométricos para a relação fantasia (FS) e orientação cultural coletivista, ficando aquém do esperado pela literatura.

TABELA 1.

Indicadores psicométricos do modelo teórico da empatia (EMRI) e orientação cultural coletivista (OCC).

\begin{tabular}{|c|c|c|c|c|c|c|c|c|}
\hline & JELOS & $\chi^{2} / g \mid$ & GFI & AGFI & $\mathrm{NFI}$ & CFI & RMR & RMSEA \\
\hline \multirow{2}{*}{ Modelo geral } & EMRI - OCC & 2.10 & 0.99 & 0.97 & 0.98 & 0.99 & 0.04 & 0.05 \\
\hline & & & & & & & & $(0.03-0.07)$ \\
\hline \multirow{8}{*}{$\begin{array}{c}\text { Modelos } \\
\text { específicos }\end{array}$} & CE-OCC & 2.05 & 0.99 & 0.96 & 0.97 & 0.98 & 0.03 & 0.04 \\
\hline & & & & & & & & $(0.03-0.07)$ \\
\hline & TP-OCC & 2.84 & 0.98 & 0.96 & 0.95 & 0.97 & 0.04 & 0.05 \\
\hline & & & & & & & & $(0.02-0.07)$ \\
\hline & AngP-OCC & 2.32 & 0.97 & 0.95 & 0.94 & 0.97 & 0.04 & 0.05 \\
\hline & & & & & & & & $(0.04-0.08)$ \\
\hline & FS-OCC & 2.55 & 0.97 & 0.93 & 0.91 & 0.94 & 0.05 & 0.06 \\
\hline & & & & & & & & $(0.04-0.09)$ \\
\hline
\end{tabular}

Notas: $p<0,05$; Consideração Empática (CE), Angústia Pessoal (AngP) e Tomada de Perspectiva (TP) e Fantasia (FS). OCC = Orientação Cultural Coletivista. 
Os pesos (saturações) que explicam 0 modelo da variável considerada na empatia e suas dimensões (CE, TP, AP, FS) sobre 0 tipo de orientação cultural coletivista (Cooperador, que colabora; Cumpridor com os demais, servidor) associaram-se, positivamente entre si (ver tabela 2). Todas as saturações (Lambdas, $\lambda$ ) estiveram dentro do intervalo esperado $|0-1|$, denotando não haver problemas de estimação proposta, pois todas estiveram estatisticamente diferentes de zero $(t>$ 1,96, $p<0,05)$. Deve-se destacar que, especificamente, a dimensão da fantasia empática apresentou um lambda associativo inferior em relação a orientação coletivista quando comparado às outras variáveis; sendo assim, sugere maior cuidado quando se pretender afirmar a influência da fantasia sobre essa orientação.

TABELA 2.

Associações lambdas da empatia e suas variações com a orientação cultural coletivista.

\begin{tabular}{lc}
\hline \multicolumn{1}{c}{ MODELOS } & $\lambda$ \\
\hline Modelo geral \\
EMRI-OCC & 0.30 \\
\hline Modelos específicos \\
CE-OCC \\
TP-OCC & 0.47 \\
AngP-OCC & 0.34 \\
FS-OCC & 0.25 \\
\hline Notas: $p<0,05 ;$ Consideração Empática (CE), Angústia Pessoal, (AngP) \\
e Tomada de Perspectiva (TP) e Fantasia (FS). OCC = Orientação \\
Cultural Coletivista.
\end{tabular}

Efetuou-se semelhante cálculo estatístico para verificar a direção deste modelo em relação a orientação individualista (ver tabela 3). Seguindo a mesma perspectiva metodológica e estatística, com base na hipótese levantada para essas variáveis, observou-se que 0 modelo que se pretendia verificar apresentou indicadores psicométricos que, também, estiveram dentro do intervalo aceito na literatura (Hair, Anderson, Tatham \& Black, 2005; Joreskög \& Sörbom, 1989) corroborando a associação da empatia em relação ao tipo de orientação cultural individualista.

TABELA 3.

Indicadores psicométricos do modelo teórico da empatia (EMRI) e orientação cultural coletivista (OCI).

\begin{tabular}{|c|c|c|c|c|c|c|c|c|}
\hline \multicolumn{2}{|c|}{ MODELOS } & $\chi^{2} / g 1$ & GFI & AGFI & $\mathrm{NFI}$ & $\mathrm{CFI}$ & RMR & RMSEA \\
\hline \multirow{2}{*}{ Modelo geral } & EMRI - OCl & 2.74 & 0.98 & 0.95 & 0.97 & 0.98 & 0.06 & 0.07 \\
\hline & & & & & & & & $(0.04-0.10)$ \\
\hline \multirow{8}{*}{$\begin{array}{c}\text { Modelos } \\
\text { específicos }\end{array}$} & CE-OCI & 2.69 & 0.98 & 0.96 & 0.93 & 0.97 & 0.05 & 0.04 \\
\hline & & & & & & & & $(0.02-0.07)$ \\
\hline & TP-OCl & 2.65 & 0.98 & 0.97 & 0.94 & 0.98 & 0.05 & 0.04 \\
\hline & & & & & & & & $(0.00-0.06)$ \\
\hline & AngP-OCl & 2.26 & 0.99 & 0.97 & 0.95 & 0.98 & 0.05 & 0.03 \\
\hline & & & & & & & & $(0.00-0.07)$ \\
\hline & $\mathrm{FS}-\mathrm{OCl}$ & 2.99 & 0.96 & 0.92 & 0.87 & 0.90 & 0.10 & 0.09 \\
\hline & & & & & & & & $(0.05-0.09)$ \\
\hline
\end{tabular}

Notas: $p<0,05$; Consideração Empática (CE), Angústia Pessoal (AngP), Tomada de Perspectiva (TP) e Fantasia (FS); OCI = Orientação Cultural Individualista. 
Os pesos (saturações) que explicam 0 modelo teórico da empatia e suas dimensões (CE, TP, AP, FS) sobre o tipo de orientação cultural individualista (Um ser único, diferente dos demais; Orientado ao êxito, ao triunfo) associaram-se, positivamente, porém, tendo lambdas fracos (ver tabela 4). Todas as saturações (Lambdas, $\lambda$ ) estivaram dentro do intervalo esperado $\mid 0$ - 1|, condição que demonstra não haver problemas de estimação proposta, pois, foram estatisticamente diferentes de zero $(t>1,96, p<0,05)$.

TABELA 4.

Associações lambdas da empatia e suas variações com a orientação cultural coletivista.

\begin{tabular}{lc}
\hline \multicolumn{1}{c}{ MODELOS } & $\lambda$ \\
\hline $\begin{array}{l}\text { Modelo geral } \\
\text { EMRI-OCl }\end{array}$ & 0.13 \\
\hline Modelos específicos & \\
CE-OCl & 0.17 \\
TP-OCl & 0.14 \\
AngP-OCl & 0.16 \\
FS-OCl & 0.10 \\
\hline Notas: $p<$ 0,05; Consideração Empática (CE), Angústia \\
PessoalV(AngP) e Tomada de Perspectiva (TP) e Fantasia \\
(FS); OCl = Orientação Cultural Individualista.
\end{tabular}

A partir desses resultados, em crítica ao modelo proposto por Formiga e Souza (2012), realizando o caminho contrário ao proposto pelos autores, corrobora-se a hipótese de que a empatia influencia as orientações culturais (isto é, aos padrões convencionais adotados por cada pessoa na sociedade e que permite as elas, priorizarem atributos dos valores culturais); isto poderá ser reconhecido, não somente a partir dos lambdas associativos, os quais apresentaram uma lógica de direção e força correspondidas a teoria, mas, também, devido aos indicadores psicométricos relacionado aos modelo teórico (por

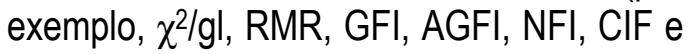
RMSEA).

De acordo com os autores supracitados, o modelo seria o contrário; a condição crítica é que as suas hipóteses não convergem com a perspectiva teórica da empatia desenvolvida por Davis (1983), o qual defende uma base psicogenética e evolutiva, podendo com isso, considerando os achados neste estudo, influenciar no decorrer do desenvolvimento psicossocial das pessoas um determinado tipo de orientação cultural a aderir, especialmente, em termos da tendência cooperadora e de interpessoalidade para a manutenção de relações sociais consistentes e permanentes para compartilhar os mesmos interesses.

Não somente a empatia, tomada como um construto único, contemplando todas as suas dimensões (Consideração Empática, Angústia Pessoal, Tomada de Perspectiva e Fantasia), mas também, com cada dimensão, foi capaz de orientar os sujeitos para agir de forma coletivista, isto é, aquela pessoa que, provavelmente, tenderia à cooperação e ao cumprimento com relação aos demais, mantendo as relações entre si e o outro de forma mais forte e segura, compartilhando os mesmos interesses; por outro lado, no desenvolvimento da empatia, não se trata apenas de inibir atributos individualistas - salientando a pessoa que prioriza o excesso do sucesso e valorização exclusiva da própria intimidade e tem 0 objetivo de obter recompensas e não se incomodar com os outros - refletindo a respeito das associações estatísticas entre estas variáveis com menor força.

Os achados no presente estudo vai na direção contrária aos defendidos por Formiga e Souza (2012), especificamente, no que se refere ao caminho teórico e metodológico proposto por esses autores entre as variáveis abordadas, isto é, a orientação cultural seria influenciadora da empatia. A que se deve tal reflexão crítica e proposta de nova testagem do modelo? Acredita-se que a orientação - seja de individualismo ou coletivismo - não ocorre no vazio, mas, estaria associado a um 
critério desenvolvimentista da empatia; este construto, com base na perspectiva de Davis (1983), pois, para assimilar as habilidades empáticas, 0 sujeito não somente deverá ser socializado nestas habilidades, mas, que, poderá desenvolver tanto um conjunto dessas habilidades (Consideração Empática, Angústia Pessoal, Tomada de Perspectiva e Fantasia) de forma independente, pois, este construto, passa pela avaliação que o sujeito faz das experiências afetivas e cognitivas que 0 conduz a uma espécie de busca pelo respeito, compreensão do outro e participação no espaço sócio-cognitivo do observador no campo dos problemas do outro, podendo promover agregação, cuidado, solidariedade, etc. (Eisenberg \& Strayer, 1990; Sampaio, Guimarães, Camino, Formiga e Menezes, 2011; Sampaio, Monte, Camino \& Roazzi, 2008).

Mas, para que tal condição ocorra, o sujeito precisaria estar disposto às aberturas do espaço interpessoal e afetivo estimulando e/ou simulando convicções, desejos, percepções, sentimentos, etc. que permita a ele se colocar no lugar do sentimento e emoção do outro, porém, não sendo o outro (Formiga, 2012); isto somente seria possível, com base nesses resultados, se e somente se, o desenvolvimento e assimilação da empatia contribuíssem para a orientação cultural de coletivismo, já que este construto valoriza a harmonia social e preservação do grupo e tradições.

Obviamente, 0 desenvolvimento da empatia não impediria as orientações individualistas, já que ambas as orientações - coletivismo e individualismo - segundo Sinha e Tripathi (1994), não devem ser consideradas opostas entre si, pois às pessoas poderão apreender uma ou outra forma dessas orientações a partir do contexto ou situação em que estiverem inseridas, a qual, para esses autores, definirá o estilo mais apropriado de conduta. O desenvolvimento da empatia seria, então, uma condição crucial para que a pessoa priorizasse melhor o tipo de orientação especialmente, a coletivista - que assumiria nas relações quando no momento de se auto-perceber e perceber os relacionamentos interpessoais.

A proposta de um modelo teórico parcimonioso, diz respeito à concentração teórico-empírica por meio da teoria da empatia, desenvolvida por Davis (1983) como influência da orientação cultural, contribuindo para que as pessoas se guiem por meio da perspectiva cultural com vista a uma sociedade mais integrada e preocupada com o outro e o seu entorno social, ao invés da valorização de condutas mais egoístas e que preze somente a si mesmo. Ao gerar esse modelo, rediscute a concepção de Formiga e Souza (2012), quando se pretende elaborar uma intervenção para a mudança de comportamento a partir da orientação cultural que os jovens atualmente se guiam; deve-se intervir nas dimensões da empatia, especialmente, na consideração empática e na tomada de perspectiva, pois, a partir da qualificação das habilidades com elas, provavelmente, 0 sujeito passaria a se orientar mais de forma coletivista do que individualista. Porém, não quer dizer que a utilização das outras dimensões empáticas de angustia pessoal e fantasia não seria útil, poderiam também causar um bom efeito, apenas, deve-se tomar cautela - já que estas apresentaram indicadores psicométricos menores em relação às outras dimensões - quando e onde seria 0 melhor momento para aplicar cada uma dessas dimensões empáticas na dinâmica jovens-comportamento-sociedade.

\section{CONSIDERAÇÕES FINAIS}

Frente a uma sociedade que, cada vez mais, investe em um 'excessivo consumo' relacional dos outros, tendo-lhes como objetos ou coisas, desvalorizando as relações consistentes e duradouras, desenvolver um estudo com foco na empatia é resgatar não somente a 
importância de se refletir sobra a humanidade, mas, apontar em direção da necessidade de que, mesmo sobre a égide da ciência, estudiosos e pessoas comuns, formem-se tanto nos conhecimentos dos fatos quanto na compreensão destes como suportes afiliativos aos outros, compreendendo a qualidade particular de um ser humano, individualmente, e distingui-lo de outro, simpatizando-se e gerando um sistema de valor do ser humano alcançando um grau de autoconhecimento do ser humano, estando atento aos estágios de crescimento social de si e da sociedade a fim de não cair no isolamento do outro e do entorno social, quanto ao direito humano e de ser humano (Berlin, 1997).

Por fim, espera-se que os objetivos deste estudo tenham sido cumpridos, principalmente, no que diz respeito comprovação do modelo causal parcimonioso entre a empatia e o tipo de orientação cultural em brasileiros, este poderá ser empregado em áreas de estudo que cooperam com a psicologia, por exemplo, educação, sociologia, serviço social, etc. Apesar dos resultados serem satisfatórios, faz-se necessário, para maior qualidade do estudo com essas variáveis, ter em conta aspectos mais específicos ou universais de cada contexto, quando se pretender avaliar tais variáveis em outras culturas (Muenjohn \& Armstrong, 2007; Triandis e cols., 1993; Triandis, 1994; Van De Vijver \& Leung, 2011). Com isso, aponta-se para a seguinte direção:

- conhecer os aspectos que podem ser comuns a todas as culturas e aqueles que são específicos, contribuindo para consolidar um marco teórico da empatia e o tipo de orientação cultural, já que, considerando os resultados encontrados é possível que ambas as variáveis venham se diferenciar entre si devido aos contextos sociais, econômicos e políticos.
- o que fazer no futuro em relação aos estudos com essas escalas? Pretende-se reunir evidências adicionais da consistência intra, inter e pan-culturais em relação a estas medidas em um modelo teórico, bem como, a realização estudos que avaliasse além das experiências empáticas, as dimensões da empatia da tomada de perspectiva do outro, fantasia, consideração empática e angustia Pessoal e sua associação com as orientações culturais em diferentes contextos educacionais e sóciodemográfico; outra condição importante para futuros estudos refere-se, relacionado ao conjunto dessas variáveis, a realização de uma análise de variância, controlando o gênero e a idade; por fim, outro estudo importante seria 0 de avaliar a convergência dessas variáveis na dinâmica familiar e escolar, colocando pais, professores e jovens para responder o mesmo instrumento a fim de comparar 0 processo socializado e sua transmissão não formação da empatia e orientação cultural em jovens. 
REFERENCIAS

Associação nacional de pesquisa e pósgraduação em psicologia - ANPEPP (2011). Contribuições para a discussão das Resoluções CNS n'. 196/96 e CFP $N^{\circ}$ 016/2000. Recuperado em 02 de Setembro de 2011, da WEB (página da WEB):http://www.anpepp.org.br/XIISim posio/Rel_ComissaoEticasobre_Res_C NS_e_CFP.pdf2000.

Atteslander, P. (1999). Social Change, Development and Anomie. In: Peter Atteslander, Bettina Gransow \& John Western (Org). Comparative anomie research: hidden barriers - hidden potential for social development. Sidney: Ashgate.

Batson, C. D.; Eklund, J. H.; Chermok, V. L.; Hoyt, J. L. \& Ortiz, B. G. (2007). An additional antecedent of empathic concern: valuing the welfare of the person in need. Journal of Personality and Social Psychology, 93 (1), 65-74.

Batson, D. C.; Tricia, R. K.; Highberger, L. \& Shaw, L. L. (1995). Immorality From Empathy-Induced Altruism: When Compassion and Justice Conflict. Journal of Personality and Social Psychology, 68 (6), 1042-1054.

Bauman, Z. (1998). O Mal-Estar da PósModernidade. 1.ed. Rio de Janeiro: Jorge Zahar Ed.

Bilich, F.; Silva, R.; Ramos, P. (2006). Análise de flexibilidade em economia da informação: modelagem de equações estruturais. Revista de Gestão da Tecnologia e Sistemas de Informação, 3 (2), 93-122.

Bürger, P. (1988). O declínio da era moderna. Revista Novos Estudos CEBRAP, 20, 81-95.

Byrne, B. M. (1989). A primer of LISREL: Basic applications and programming for confirmatory factor analytic models. New York: Springer-Verlag.

Conselho Nacional de Saúde - CNS. (1996). Diretrizes e Normas Regulamentadoras de Pesquisas Envolvendo Seres Humanos. Recuperado em 02 de Setembro de
2011, da WEB (página da WEB): http://conselho.saude.gov.br/resolucoe s/reso_96.htm.

Davis, M. H. (1983). Measuring individual differences in empathy: Evidence for a multidimensional approach. Journal of Personality and Social Psychology, 44, 113-126.

Decety J.; Michalska K. J. \& Akitsuki, Y. (2008). Who caused the pain? A functional MRI investigation of empathy and intentionality in children. Neuropsychologia. 46, 2607-2614.

Decety, J. \& Jackson, P. L. (2004). The functional architecture of human empathy. Behavioral and Cognitive Neuroscience Reviews. 3, 71-100.

Decety, J. (2005). Perspective taking as the royal avenue to empathy. In B. F. Malle e S. D. Hodges (Eds.), Other minds: How humans bridge the divide between self and other. (pp. 143-157). New York: Guilford Publications.

Eisenberg, N. \& Strayer, J. (1990). Empathy and its development. New York: Cambridge University Press.

Enz, N. \& Zoll, N. (2006). Cultural differences in empathy between China, Germany and the UK. Recuperado em 23 de novembro de 2006, de www.nicve.salford.ac.uk/elvis/ resources/empathy.

Formiga, N. S. (2012). Um estudo intracultural da consistência estrutural da escala multidimensional de reatividade interpessoal (EMRI). Revista salud y sociedad, 3 (3), 251-26.

Formiga, N. S. (2004). 0 tipo de orientação cultural e sua influência sobre os indicadores do rendimento escolar. Psicologia. Teoria e Prática, 16 (1), 1329.

Formiga, N. S.; Camino, C. \& Galvão, L. (2009). Empatia, desenvolvimento moral e conduta desviante em adolescentes: testagem de um modelo teórico. In: VII Congresso Brasileiro de Psicologia do Desenvolvimento, 2009, Rio de Janeiro. (pp. 541-542). Rio de Janeiro, RJ: CBPD. 
Formiga, N. S. \& Diniz, A. (2011). Estilo da orientação cultural e condutas desviantes: Testagem de um modelo teórico. Revista Pesquisa em Psicologia, 5 (1), 2-11.

Formiga, N. S.; Sampaio, L. R.; Guimaraes, P. R. B. \& Camino, C. P. S (2011). Escala multidimensional de reatividade interpessoal de Davis: Quatro ou três fatores mensuram a empatia? Artigo submetido à avaliação.

Formiga, N. S. \& Souza, M. A. (2012). Tipo de orientação cultural e empatia em brasileiros: Verificação de um modelo teórico. Estudos Interdisciplinares em Psicologia, 3 (2), 139-161.

Garson, G. D. (2003). PA 765 Statnotes: An online textbook. Endereço de página Web:

http://www2.chass.ncsu.edu/garson/pa 765/statnote.htm (consultado dia 17 de maio de 2005).

Gouveia, V. V.; Clemente, M. \& Vidal, M.A. (1998). España desde dentro: el individualismo y el colectivismo como rasgos diferenciadores de las comunidades autónomas. Sociedade y Utopia, 11, 168-179.

Hair, J. F.; Anderson, R. E.; Tatham, R. L.; Black, W. (2005). Análise Multivariada de Dados. Porto Alegre: Bookman.

Hoffman, M. L. (2000). Empathy and moral development: Implications for caring and justice. New York: Cambridge University Press.

Hofstede, G. (1980). Culture's consequences. Beverly Hills, CA: Sage.

Inglehart, R. (1991). El cambio cultural en las sociedades industriales avanzadas. Madrid: Centro de Investigaciones Sociológicas / Siglo XXI Editores.

Jodelet, D. (1984). Représentation sociale: phénomènes, concept et théorie. In: $\mathrm{S}$. Moscovici (Ed.) Psychologie Sociale, (pp. 357-378). Paris: Presses Universitaires de France.

Joreskög, K. \& Sörbom, D. (1989). LISREL 7 user's reference guide. Mooresville: Scientific Software.
Kelloway, E. K. (1998). Using LISREL for structural equation modeling: $A$ researcher's guide. Thousand Oaks, CA: Sage Publications.

Kumar, K. (1997). Da sociedade pósindustrial à pós-moderna: novas teorias sobre o mundo contemporâneo. Rio de Janeiro: Zahar.

Lipovetsky, G. \& Charles, S. (2004). Os Tempos Hipermodernos. São Paulo: Barcarolla,

Mehrabian, A. \& Epstein, N. (1972). A measure of emotional empathy. Journal of Personality, 40, 525-543.

Muenjohn, N. \&Armstrong, A.( 2007). Transformational Leadership: The Influence of Culture on the Leadership Behaviours of Expatriate Managers. International Journal of Business and Information, 2 (2), 265-283.

Rokeach, M. (1973). The nature of human values. New York: The Free Press.

Rokeach, M. (1979). Introduction. Em M. Rokeach (Ed.), Understanding human values: Individual and societal. (pp. 111). New York: The Free Press.

Sampaio, L. R.; Guimarães, P. R. B.; Camino, C. P. S; Formiga, N. S. \& Menezes, I. G. (2011). Estudos sobre a dimensionalidade da empatia: tradução e adaptação do Interpersonal Reactivity Index (IRI). Psico, 42 (1), 67-76.

Sampaio, L. R.; Monte, F. C.; Camino, C. \&Roazzi, A. (2008). Justiça distributiva e empatia em adolescentes do nordeste brasileiro. Psicologia: Reflexão e Crítica, 21 (2), 275-282.

Schwartz, S. H. (1990). Individualismcollectivism: Critique and proposed refinements. Journal of Cross-Cultural Psychology, 21, 139-157.

Sinha, D. \& Tripathi, R. C. (1994). Individualism in a collectivist culture: A case of coexistence of opposites. Em U. Kim, H.C. Triandis, Ç. Kagitçibasi, S.-C. Choi \& G. Yoon (Eds.), Individualism and collectivism: Theory, method, and applications. (pp. 123136). Thousand Oaks, CA: Sage Publications. 
Teixeira, E. B. (2005). Aventura pósmoderna e sua sombra. São Paulo-SP: Paulus.

Triandis, H. C. (1995). Individualism and collectivism. Boulder, CO: Westview Press.

Triandis, H. C. (1996). The psychological measurement of cultural syndromes. American Psychologist, 51, 407-415.

Triandis, H.C., Chen, X.P. \& Chan, D. K. S. (1998). Scenarios for the measurement of collectivism and individualism. Journal of Cross-Cultural Psychology, 29, 275-289.

Trianis, H. C.; Mccusker, C.; Betancourt, H.; Iwao, S.; Leung, K.; Salazar, J. M. ; Setiadi, B.; Sinha, B. P.; Touzard, H.; Zaleski, Z. (1993). Na etic-emic analysis of individualism and collectivism. Journal of cross-cultural psychology, 24 (3), 366-383.

Van de Vijver, F. J., \& Leung, K. (2011). Equivalence and bias: A review of concepts, models, and data analytic procedures. In D. R. Matsumoto \& F. J. van de Vijver (Eds.), Culture and psychology. Cross-cultural research methods in psychology (pp. 46-70). New York: Cambridge University Press.

Wispé, L. (1990). History of the concept of empathy. In: N. Eisenberg \& J. Strayer (org), Empathy and its development. (pp 17-37). New York: Cambridge University Press. 